\title{
1 Functional diversity increases the efficacy of phage combinations
}

2 Rosanna C. T. Wright ${ }^{1}$, Ville-Petri Friman ${ }^{2}$, Margaret C. M. Smith², Michael A. Brockhurst ${ }^{1}$

1. Faculty of Biology, Medicine and Health, University of Manchester, Manchester, UK

5 Abbreviations: Type IV Pilus (T4P); Lipopolysaccharide (LPS)

\section{Abstract}

7 Phage therapy is a promising alternative to traditional antibiotics for treating bacterial 8 infections. Such phage-based therapeutics typically contain multiple phages, but how the

9 efficacy of phage combinations scales with phage richness, identity and functional traits is 10 unclear. Here, we experimentally tested the efficacy of 827 unique phage combinations 11 ranging in phage richness from 1 to 12 phages. The efficacy of phage combinations increased

12 with phage richness. However, complementarity between functionally diverse phages allowed 13 efficacy to be maximised at lower levels of phage richness in functionally diverse 14 combinations. These findings suggest that phage functional diversity is the key property of effective phage combinations, enabling the design of simple but effective phage therapies that overcome the practical and regulatory hurdles that limit development of more diverse phage

17 therapy cocktails. 


\section{Introduction}

Bacterial killing by lytic phages regulates bacterial turnover in microbial communities, influencing bacterial community dynamics in both environmental and clinical settings [1, 2]. Phage diversity is predicted to exceed that of their hosts by up to 10 times [3], and may vary between communities just metres apart [4]. A survey of phages able to infect Pseudomonas aeruginosa, a commonly multi-drug resistant opportunistic pathogen [5], across 4 continents identified 7 distinct phage groups with lytic activity against $87 \%$ of clinical bacterial strains tested [6], and novel phage taxa are continually being discovered $[7,8]$. This diversity of phages offers a promising alternative to antibiotics for treating bacterial infection where rates of antibiotic resistance are rapidly rising [9-11]. Multiple phages are often combined for therapeutic use to improve the range of hosts which can be targeted. However, more diverse combinations pose greater regulatory hurdles as individual phages and interaction effects must be assessed [12-14]. While developing efficient, low diversity phage combinations would be highly practical, it is unclear which rationale one should use to design such combinations.

The relationship between biodiversity and ecosystem functioning (i.e., the collective activity of a community) is usually positive [15]. Increasing species richness has been shown to improve the function of microbial communities by two distinct mechanisms [16, 17]. Firstly, if species perform different ecological roles, then greater species richness can deliver higher communitylevel performance due to functional complementarity, through filling more of the available niche space $[16,18]$. Secondly, more diverse communities are more likely to contain highly performing taxa simply by chance, leading to a positive relationship between diversity and function due to species identity effects $[16,17]$. On the other hand, functional redundancy among species in a community can lead to diminishing returns of further increasing species richness, resulting in a saturating relationship between richness and function [19-21]. These counteracting effects suggest that high phage efficacy could be attained by low richness

43 phage combinations, provided that these contain functionally different and non-redundant 44 phages. Such combinations would have practical benefits in terms of reducing the 
manufacturing and regulatory challenges posed by higher order phage combination therapies $[13,14,22]$

47 For phages, lytic infection of a bacterial host depends on adsorption to the host outer membrane and evasion of host phage defence systems once within the cell [23]. Binding to specific bacterial cell-surface receptors for adsorption is therefore a key functional trait for phages. Phage combinations targeting higher numbers of receptors could be considered

51 functionally diverse and more efficacious by reducing competition among phages for shared 52 adsorption sites. Such phage combinations could also limit resistance evolution via cell surface modification since this would likely require multiple mutations [24-26], that often impose additive fitness costs [27].

By applying the principles of biodiversity-ecosystem functioning to the design of phage therapies, we sought to determine the relative importance of phage species richness,

57 functional diversity and identity effects on the efficacy of phage combinations. We used twelve Pseudomonas aeruginosa phages including phages targeting either lipopolysaccharide (LPS) or Type IV pilus (T4P) for adsorption to result in 827 unique phage combinations with differing levels of species richness and degrees of functional diversity. These combinations included all possible single, pairwise and 3-member communities, 264 different 4- and 6-member communities and the full 12-member community. We show that phage richness had a saturating relationship with efficacy (defined as the suppression of bacterial growth), and that highly efficacious but low richness phage combinations could be designed provided that they had high functional diversity, i.e., the constituent phages targeted multiple distinct adsorption receptors. Together, these results suggest that ecological complementarity plays a key role in determining the efficacy of phage combinations. 
70 A panel of 12 lytic $P$. aeruginosa phages were used to build phage combinations of varying

71 phage community species richness. The adsorption receptors of all phages has previously

72 been characterised [28]; 4 phages adsorb via T4P, and 8 via LPS. In total, we assembled 827

73 different phage combinations, ranging from single phage (12), all possible 2- and 3-member

74 communities (66 and 220 respectively), a random partition of 4 - and 6-member communities

75 (264 of each), to the full 12-member community. A random partition design was used to select

764 and 6-phage communities which equally represent all phage strains across both richness

77 levels (as described previously [29]).

Phage stocks were amplified to equal densities $\left(\sim 8.9 \times 10^{10} \pm 1.0 \times 10^{11} \mathrm{pfu} / \mathrm{ml}\right)$ using the susceptible bacterial host $P$. aeruginosa PAO1, isolated by filtration $(0.22 \mu \mathrm{m})$ and stored at $4^{\circ} \mathrm{C}$. To limit human error, master plates of phage communities were assembled in deep 96 well plates using a liquid handling robot (epMotion ${ }^{\circledR} 5070$, Eppendorf, Germany) in triplicate.

82 Equal volumes (and densities) of each phage were added to give a final volume of $120 \mu \mathrm{l}$ per phage community.

We determined efficacy of phage combinations as their ability to supress growth of the susceptible host, $P$. aeruginosa PAO1. Bacterial replicates were inoculated from three single colonies into $6 \mathrm{ml} \mathrm{KB}$ and grown overnight at $37^{\circ} \mathrm{C}$ with shaking at $180 \mathrm{rpm}$, before diluting $100-$ fold into assay plates containing $120 \mu \mathrm{l}$ of $\mathrm{KB}$. Phage communities were transferred from the master plates $(15 \mu \mathrm{l}$ per well) to give a multiplicity of infection of approximately 100 phage per bacterial cell (actual $\mathrm{MOI} \sim 80.4 \pm 20.2$ with initial bacterial density of $\sim 9.6 \times 10^{7} \pm 1.1 \times 10^{7}$ ). Optical density (absorbance at $600 \mathrm{~nm} ; A b s_{600}$ ) was measured immediately, then after static $24 \mathrm{~h}$ incubation at $37^{\circ} \mathrm{C}$. Phage combination efficiency was measured as reduction in bacterial growth in the presence relative to the absence of phage, as 'Efficacy' (Eq 1; [30]).

$$
\text { Efficacy }=1-\frac{\left[A b s_{600}(t=24 h)-A b s_{600}(t=0 h)\right]_{\text {phage }}}{\left[A b s_{600}(t=24 h)-A b s_{600}(t=0 h)\right]_{\text {phage }^{-}}}
$$


95 For each phage combination, the phage with the highest independent Efficacy value was

96

97 model as it was not significant. Non-linear richness was included as an explanatory variable, considered as the best constituent phage (i.e., measured when Diversity $=1$; matched by replicate to the phage combination). These values were used to calculate transgressive overyielding, $D_{\max }$ (Eq. 2; [31]), which is a measure that describes the efficacy of a community relative to its most efficient member, such that a value of 0 indicates equal efficacy and values above 0 indicates that the community was more effective.

\section{Statistical analysis}

The relationship between phage strain richness and efficacy was analysed using a previously described linear model method designed to separate significant factors affecting biodiversityecosystem functioning [29]. Phage richness and functional diversity (i.e., number of different receptor targets) were included as interacting main effects, alongside other main effects of receptor targets (i.e., presence of LPS and/or T4P targeting phages), phage identity (including pairwise and higher order interactions between phages as separate main effects) and nonlinear effects of phage richness. Due to the saturating relationship between phage richness and efficacy, we also fitted a non-linear asymptotic exponential model to the data. Model parameters were determined using the nonlinear least squares function in $R$ [32], and compared to equivalent linear models using Akaike Information Criterion (AIC).

The effects of richness, functional diversity and phage identity on transgressive overyielding were also analysed using a linear model. Significant interaction terms between functional diversity and richness, functional diversity and phage identity, and pairwise interactions between phages were included in the model. Here, receptor target was excluded from the and best fit of regression models (linear versus decaying exponential model) were assessed 
119 by AIC to fit regression curves to the plotted data. All analyses were performed in R (version

$120 \quad 3.5 .2 ;[32])$.

\section{Results}

122 Diminishing returns of increasing phage richness on phage combination efficacy

123 The efficacy of phage combinations, measured as their ability to reduce bacterial growth,

124 increased with phage community richness (i.e., number of phage strains). However, phage

125 richness explained only $30 \%$ of variation in efficacy (Figure 1 ; linear model efficacy: $_{1,5740}=2497$,

$\left.126 \mathrm{p}<0.0001, \mathrm{R}^{2}=0.303\right)$. Non-linear models explained a greater proportion of the variation: the

127 relationship between richness and efficacy was best explained by an asymptotic exponential

128 model where the asymptote was reached when bacterial growth was completely suppressed

129 (Figure 1; AIC linear model $=205 ;$ AIC asymptotic model $=-1190$ ). This suggests that there were low

130 richness phage combinations that were as effective as the highest richness phage

131 combination, whose efficiency could not be improved with additional phages, likely due to

132 functional redundancy among the constituent phage strains.

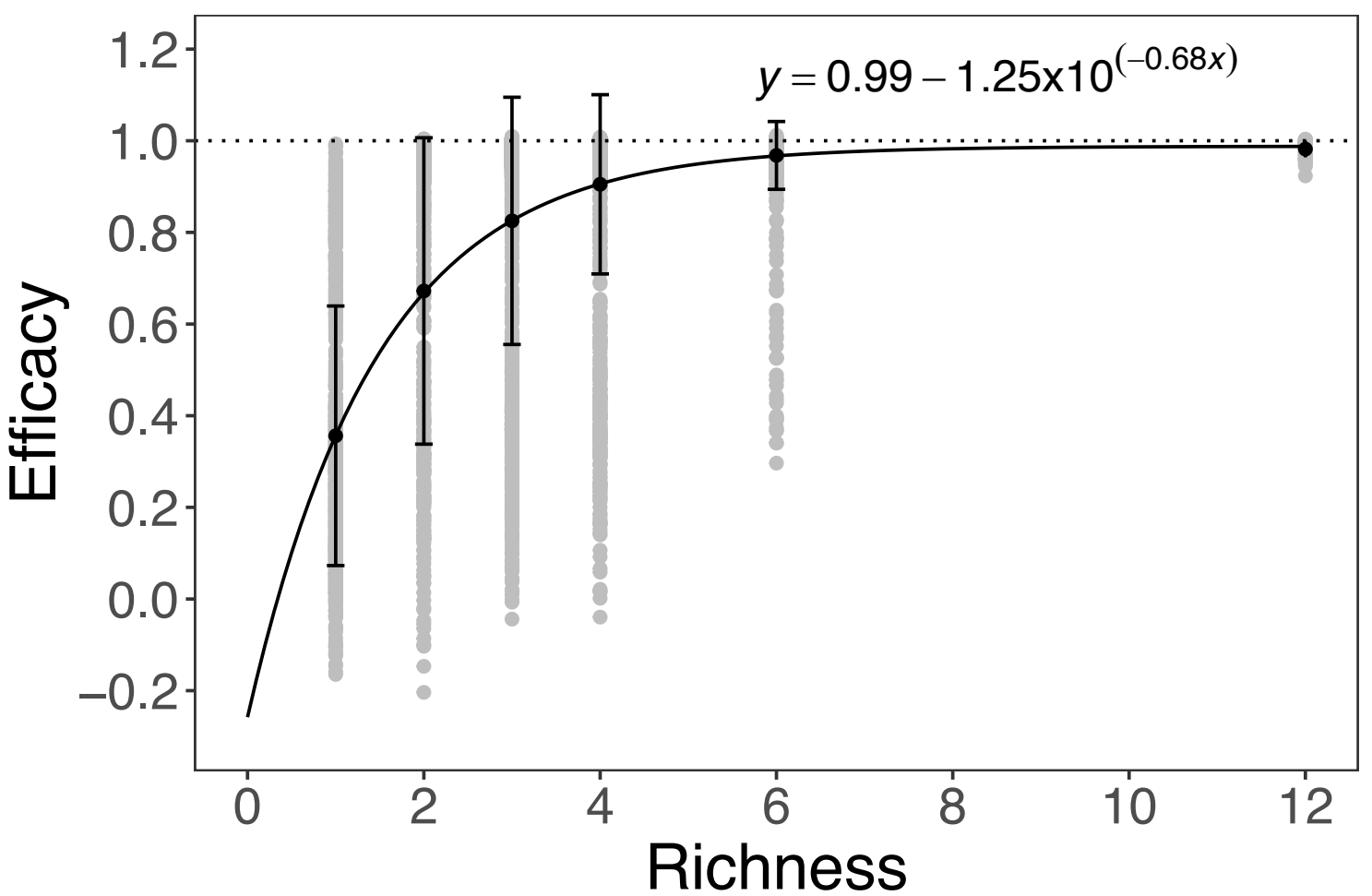


134 Figure 1. Saturating relationship between the efficacy and richness of phage

135 combinations. Efficiency of phage combinations measured as mean efficacy $( \pm$ s.d.) of

136 bacterial growth suppression in the presence of phage relative to phage-free growth, raw data

137 in grey. The dashed line at 1 indicates complete suppression of bacterial growth by the phage

138 community. An asymptotic exponential with the equation shown was fit to the data using a

139 non-linear least squares model.

140 Increased phage functional diversity improves phage combination efficacy

141 Including functional diversity (FD), in terms of the receptor targeted by the phage, in the model

142 explained substantially more of the observed variation in efficacy: phage functional diversity

143 and strain richness together accounted for $\sim 70 \%$ of the variation in efficacy (Figure 2; linear

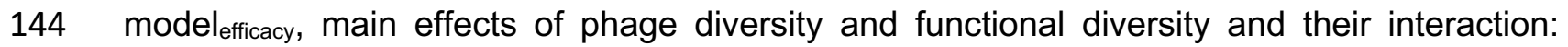

$\left.145 F_{3,5738}=4476, p<0.0001, R^{2}=0.701\right)$. Phages targeting T4P contributed more to efficacy than

146 LPS-binding phages, such that combinations containing only T4P-binding phages

147 outperformed combinations containing only LPS-binding phages (Figure 2A+B; linear

148 model $_{\text {efficacy, }}$ coefficient of T4P-binding phages: $t=11.16, p<0.0001$; coefficient of LPS-binding

149 phages: $\mathrm{t}=(-) 24.57, \mathrm{p}<0.001)$. Functionally diverse combinations containing phages targeting

150 both LPS and T4P receptors showed a saturating relationship between efficacy and phage

151 richness and completely supressed bacterial growth at lower levels of phage diversity than

152 combinations targeting only a single receptor (Figure 2C). Phage identity accounted for only

$153 \sim 3 \%$ of the remaining variation in efficacy (linear model efficacy $_{\text {: }} F_{12,5730}=15.45, p<0.0001$ ).

154 Consistent with stronger complementarity effects at higher functional diversity, we observed

155 greater transgressive overyielding for high compared to low functional diversity combinations

156 (Figure 3; linear model $D_{D \max }, \mathrm{F}_{1,5740}=498.7, \mathrm{p}<0.0001$ ). Moreover, transgressive overyielding

157 was stronger at lower phage richness levels for high but not low functional diversity

158 combinations (Figure 3 , linear model $\left.\right|_{D \max }$, richness $F_{1,5740}=31.1, p<0.0001$; interaction:

159 functional diversity * richness $\left.F_{3,5738}=136, p<0.0001\right)$. Together these data suggest that 


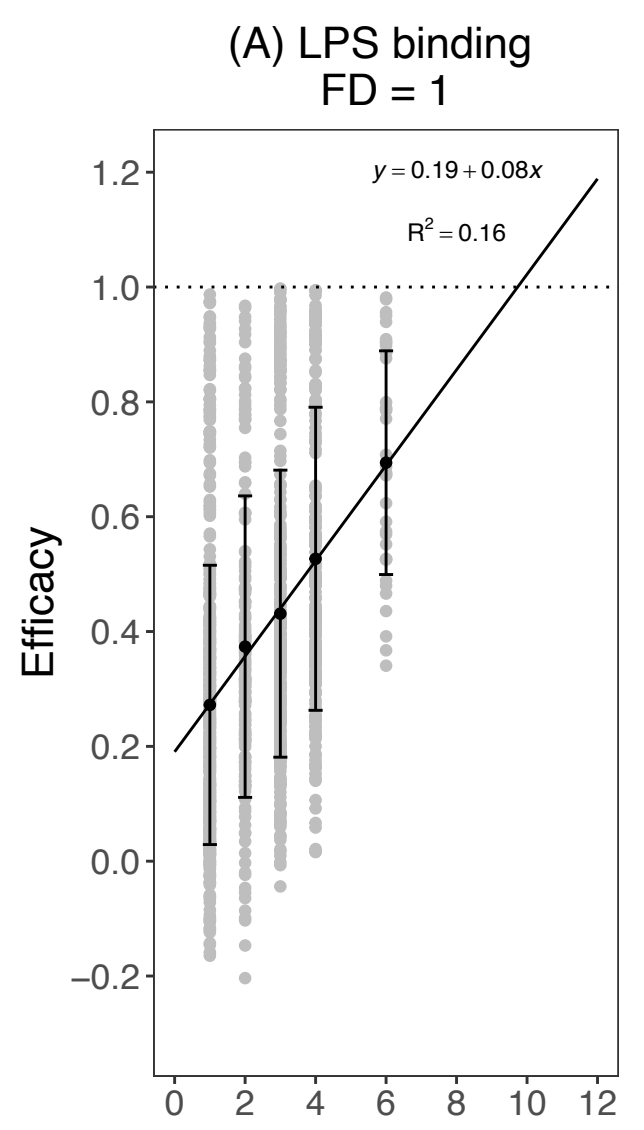

(B) T4P binding $\mathrm{FD}=1$

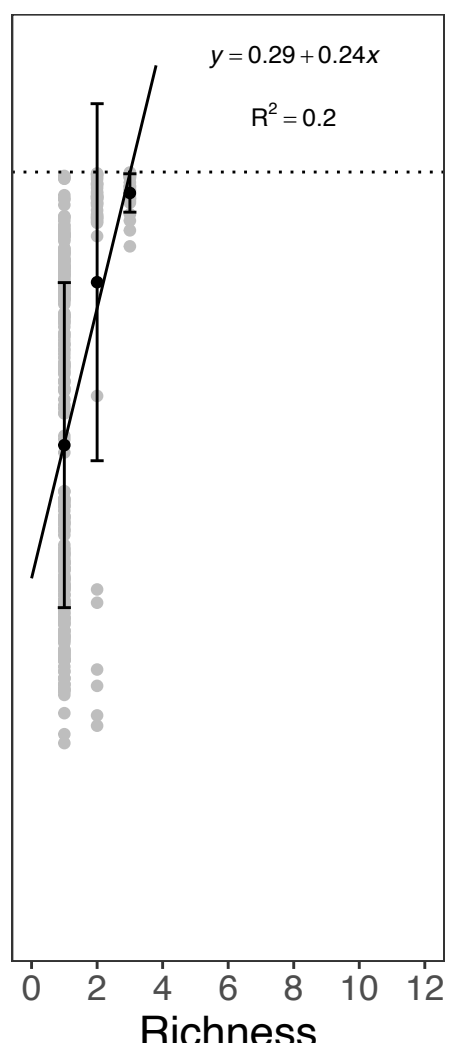

(C) LPS + T4P binding $\mathrm{FD}=2$

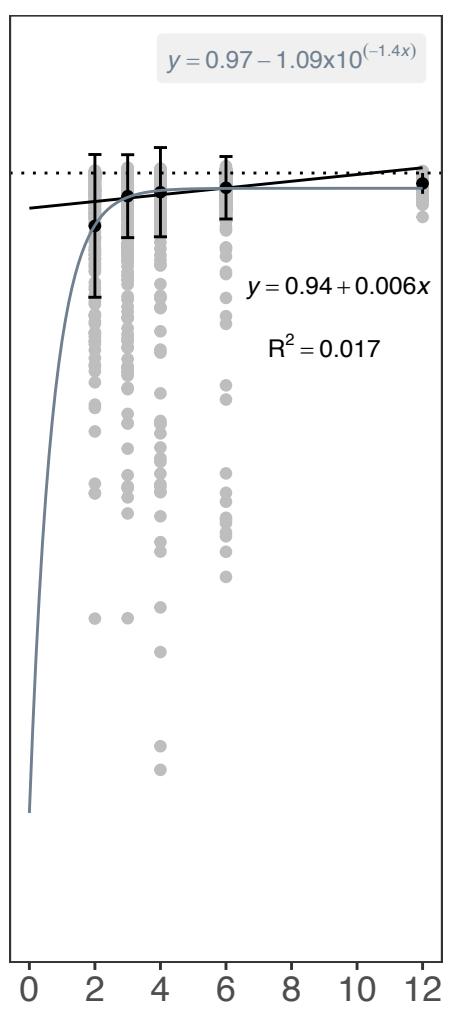

Figure 2. Functional diversity increases efficacy of phage combinations. Efficacy of phage combinations with low functional diversity $(F D=1)$, where all phage target either LPS (A) or Type IV pilus (B), and phage combinations with high functional diversity (FD = 2) which include phages targeting both the LPS and Type IV Pilus (C). Efficacy is measured as suppression of bacterial growth by phages relative to phage-free populations; mean values $( \pm$ s.d.) are shown in black, with raw data in grey to show distribution of data points. The dashed line indicates the theoretical maximum reduction (i.e., no bacterial growth detected). Linear regression equations relate to relationship between phage richness $(x)$ and efficacy $(y)$; note

171 that for (C), an asymptotic exponential model (shown in grey) better explains this relationship $\left(\mathrm{AIC}_{\text {linear model }}=205 ; \mathrm{AIC}\right.$ asymptotic model $\left.=-1190\right)$. 


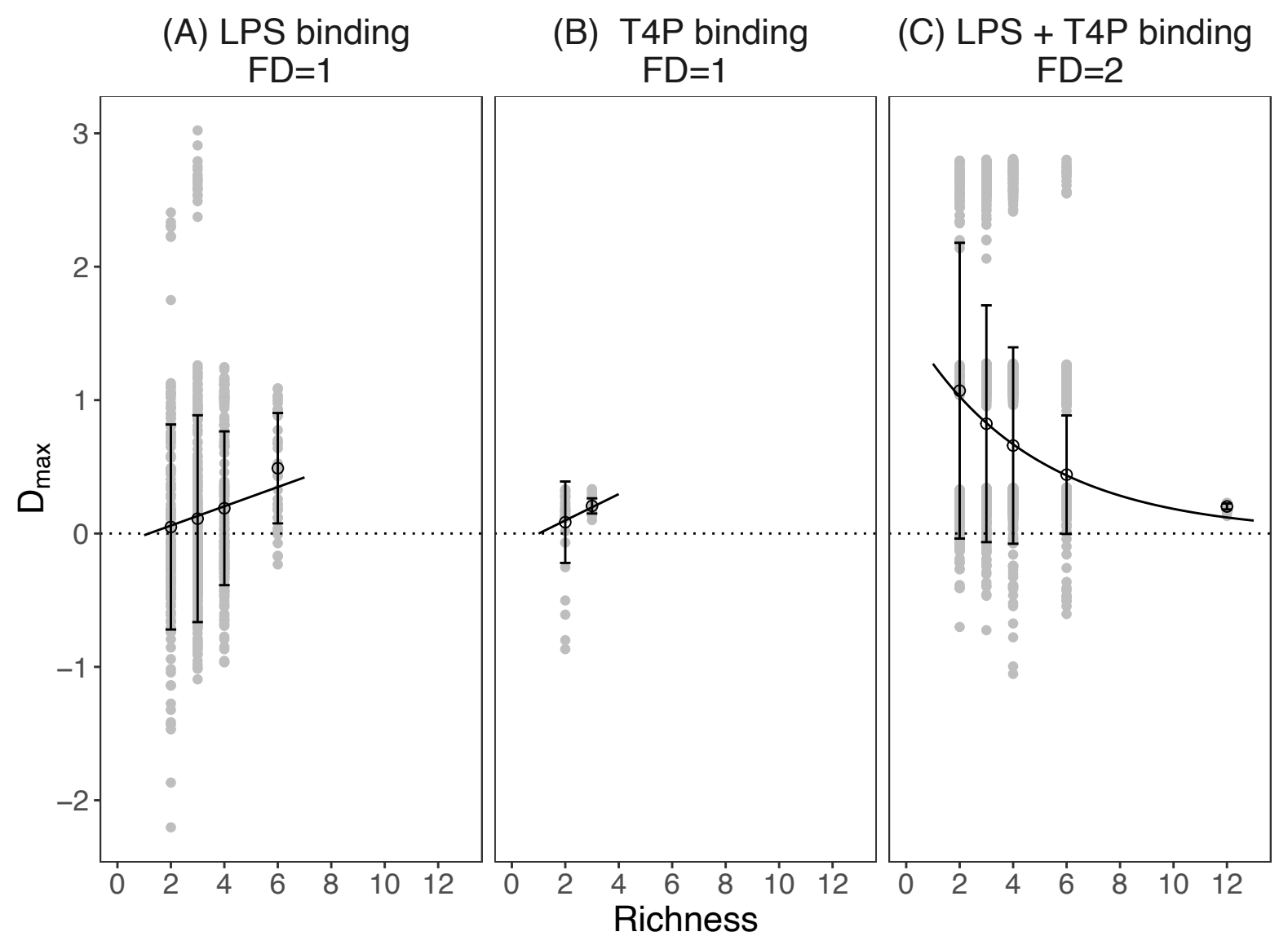

174 Figure 3. Degree of transgressive overyielding is determined by phage functional

175 diversity. Transgressive overyielding, $D_{\max }$, describes the efficacy of phage combinations

176 relative to the best constituent phage as a monoculture. Phage combinations either target

177 one receptor (FD = 1; A, LPS binding; $B$, T4P binding) or include phages targeting LPS and

178 targeting T4P (FD = 2; C, LPS + T4P binding). Mean values ( \pm s.d.) are shown in black, with

179 raw data in grey to show distribution of data points; regression lines were fit either as a linear

180 model $(A-B)$ or an exponential degradation model $(C)$.

\section{Discussion}

182 To enable rational design of phage therapy combinations, it is important to understand the key

183 factors which determine a phage combination's efficacy in supressing bacterial growth.

184 Applying concepts from the analysis of ecological biodiversity-ecosystem function 185 relationships, we compared the relative contributions of phage richness, phage identity and 186 functional diversity in determining the efficacy of phage combinations. We observed a 
saturating relationship between phage richness and efficacy, consistent with diminishing returns of increasing richness due to functional redundancy among phages at higher richness levels. Correspondingly, phage combinations with higher functional diversity, in terms of the number of cell surface receptors targeted for phage adsorption, were more effective at suppressing bacterial growth and were able to do so at lower levels of phage richness (e.g., 2-3 phages) than low functional diversity combinations. Functionally diverse phage combinations targeting different adsorption receptors displayed higher transgressive overyielding and stronger complementarity at low levels of phage richness, achieving up to 3fold higher efficacy than their best constituent phage even for combinations of just two phages. Together, our data suggest that functional diversity is the most important determinant of the efficacy of phage combinations.

By maximising functional diversity, phage combinations can be optimised for bacterial killing at low strain diversity, thus reducing the regulatory hurdles of preparing more complex therapeutic combinations $[13,14,22]$. Functional complementarity between phages targeting different adsorption receptors is likely to have two key benefits: Firstly, decreased competition for binding sites to adsorb to the bacterial cell may lead to increased lysis. Secondly, functionally diverse phage combinations are more likely to suppress resistance evolution. The majority of resistance mutations arising against our phage panel target the genes encoding the bacterial cell surface receptors (LPS and T4P; [28, 33]), and as such, promote crossresistance to alternative phages which adsorb to the same receptor. In contrast, resistance to a functionally diverse phage combination is likely require multiple independent resistance mutations (e.g. modification of each adsorption target; [33]) which will co-occur in the same cell with far lower probability.

In addition to increasing efficacy against a single bacterial genotype, higher functional diversity may also prove beneficial in more complex scenarios. For example, functionally diverse phage

212 combinations are likely to be able to target a broader diversity of bacterial genotypes. This could be particularly relevant in treatment of chronic infections, where the bacterial populations 
214 typically undergo extensive evolutionary diversification (e.g., in response to host-pathogen

215 interactions) [34, 35]. This can lead to altered expression of common phage receptor targets,

216 including modification and even loss of LPS components and T4P [36-38], which can reduce

217 susceptibility to phage infection [39, 40]. Essentiality of different cell-surface receptors across

218 environments may explain differences in observed efficacy between phages targeting different

219 adsorption receptors. Phage combinations targeting a broader range of cell surface receptors

220 will be more likely to be able to infect and clear such host-adapted bacterial populations.

221 In this study, functional phage diversity was limited to two cell surface receptor targets, but

222 further increases in the diversity of receptors targeted by phage combinations is likely to lead

223 to further increases in their efficacy. Examples of other $P$. aeruginosa cell surface receptors

224 used for phage adsorption include, outer membrane porins [41] and other membrane anchored proteins such as TonB-dependent receptors, which can be involved in ironsiderophore uptake $[24,42]$. An additional limitation of this study is that we did not test a strain encoding a CRISPR-Cas immunity system [43]. Inducible resistance mechanisms may be preferentially selected in vivo because of their lower fitness costs compared to surface receptor modification mutations [44]. Unlike surface modification resistance mutations, CRISPR-mediated resistance is likely to promote different cross-resistance interactions between phages mediated by their genetic similarity rather than their receptor target for adsorption. This suggests that whilst functional diversity of phage strains is necessary to limit

233 the evolution of cross-resistance via surface modification, maximising genetic diversity could

234 be important to limit cross-resistance via sequence-based resistance mechanisms such as CRISPR-Cas, restriction modification or other recently discovered phage defence systems [45].

237 To conclude, our findings suggest that maximising functional diversity is a simple and effective 238 rule for designing high efficacy, low richness phage combinations overcoming the regulatory 239 hurdles associated with preparation of complex phage cocktails. 


\section{Acknowledgements}

This work was supported by an ACCE DTP PhD Studentship to RCTW funded by the

Natural Environment Research Council (NE/L002450/1, Studentship 1517986). We thank

Matti Jalasvuori for providing us with phage strains.

\section{References}

245 1. Harrison F. Microbial ecology of the cystic fibrosis lung. Microbiology 2007; 153: 917923.

2. Levin BR, Bull JJ. Population and evolutionary dynamics of phage therapy. Nat Rev Microbiol 2004; 2: 166-173.

3. Clokie MR, Millard AD, Letarov AV, Heaphy S. Phages in nature. Bacteriophage 2011;

$$
\text { 1: } 31-45 \text {. }
$$

4. Frederickson CM, Short SM, Suttle CA. The Physical Environment Affects Cyanophage Communities in British Columbia Inlets. Microb Ecol 2003; 46: 348-357.

5. Breidenstein EBM, de la Fuente-Núñez C, Hancock REW. Pseudomonas aeruginosa: all roads lead to resistance. Trends Microbiol 2011; 19: 419-426.

6. Ceyssens P-J, Noben J-P, Ackermann H-W, Verhaegen J, Vos DD, Pirnay J-P, et al. Survey of Pseudomonas aeruginosa and its phages: de novo peptide sequencing as a novel tool to assess the diversity of worldwide collected viruses. Environ Microbiol 2009; 11: 1303-1313.

7. Amgarten D, Martins LF, Lombardi KC, Antunes LP, de Souza APS, Nicastro GG, et al. Three novel Pseudomonas phages isolated from composting provide insights into the evolution and diversity of tailed phages. BMC Genomics 2017; 18: 346.

8. Sepúlveda-Robles O, Kameyama L, Guarneros G. High Diversity and Novel Species of Pseudomonas aeruginosa Bacteriophages. Appl Env Microbiol 2012; 78: 4510-4515.

9. Chan BK, Abedon ST, Loc-Carrillo C. Phage cocktails and the future of phage therapy. Future Microbiol 2013; 8: 769-783. 
10. Pires DP, Boas DV, Sillankorva S, Azeredo J. Phage therapy: a step forward in the treatment of Pseudomonas aeruginosa infections. J Virol 2015; JVI.00385-15.

11. World Health Organization. Antimicrobial resistance: global report on surveillance. 2014.

12. Pirnay J-P, Blasdel BG, Bretaudeau L, Buckling A, Chanishvili N, Clark JR, et al. Quality and Safety Requirements for Sustainable Phage Therapy Products. Pharm Res 2015; 32: 2173-2179.

13. Merabishvili M, Pirnay J-P, De Vos D. Guidelines to Compose an Ideal Bacteriophage Cocktail. In: Azeredo J, Sillankorva S (eds). Bacteriophage Therapy: From Lab to Clinical Practice. 2018. Springer New York, New York, NY, pp 99-110.

14. Verbeken G, Pirnay J-P, Vos DD, Jennes S, Zizi M, Lavigne R, et al. Optimizing the European Regulatory Framework for Sustainable Bacteriophage Therapy in Human Medicine. Arch Immunol Ther Exp (Warsz) 2012; 60: 161-172.

15. Tilman D, Isbell F, Cowles JM. Biodiversity and Ecosystem Functioning. Annu Rev Ecol Evol Syst 2014; 45: 471-493.

16. Bell T, Newman JA, Silverman BW, Turner SL, Lilley AK. The contribution of species richness and composition to bacterial services. Nature 2005; 436: 1157-1160.

17. Evans R, Alessi AM, Bird S, McQueen-Mason SJ, Bruce NC, Brockhurst MA. Defining the functional traits that drive bacterial decomposer community productivity. ISME J

18. Salles JF, Poly F, Schmid B, Roux XL. Community niche predicts the functioning of denitrifying bacterial assemblages. Ecology 2009; 90: 3324-3332.

19. Petchey OL, Gaston KJ. Functional diversity: back to basics and looking forward. Ecol Lett 2006; 9: 741-758.

20. Tilman D, Knops J, Wedin D, Reich P, Ritchie M, Siemann E. The Influence of Functional Diversity and Composition on Ecosystem Processes. Science 1997; 277: $1300-1302$. 
21. Yin B, Crowley D, Sparovek G, Melo WJD, Borneman J. Bacterial Functional Redundancy along a Soil Reclamation Gradient. Appl Env Microbiol 2000; 66: 43614365.

22. Brüssow $\mathrm{H}$. What is needed for phage therapy to become a reality in Western medicine? Virology 2012; 434: 138-142.

23. Labrie SJ, Samson JE, Moineau S. Bacteriophage resistance mechanisms. Nat Rev Microbiol 2010; 8: 317-327.

24. Betts A, Gray C, Zelek M, MacLean RC, King KC. High parasite diversity accelerates host adaptation and diversification. Science 2018; 360: 907-911.

25. Gurney J, Aldakak L, Betts A, Gougat-Barbera C, Poisot T, Kaltz O, et al. Network structure and local adaptation in coevolving bacteria-phage interactions. Mol Ecol 2017.

26. Tanji Y, Shimada T, Yoichi M, Miyanaga K, Hori K, Unno H. Toward rational control of Escherichia coli O157:H7 by a phage cocktail. Appl Microbiol Biotechnol 2004; 64: $270-274$.

27. Koskella B, Lin DM, Buckling A, Thompson JN. The costs of evolving resistance in heterogeneous parasite environments. Proc R Soc Lond B Biol Sci 2011; rspb20112259.

28. Wright RCT, Friman V-P, Smith MCM, Brockhurst MA. Cross-resistance is modular in bacteria-phage interactions. PLOS Biol 2018; 16: e2006057.

29. Bell T, Lilley AK, Hector A, Schmid B, King L, Newman JA, et al. A Linear Model Method for Biodiversity-Ecosystem Functioning Experiments. Am Nat 2009; 174: 836849.

30. Poullain V, Gandon S, Brockhurst MA, Buckling A, Hochberg ME. The evolution of specificity in evolving and coevolving antagonistic interactions between a bacteria and its phage. Evolution 2008; 62: 1-11.

31. Loreau M. Separating Sampling and Other Effects in Biodiversity Experiments. Oikos 1998; 82: 600-602. 
32. R Core Team. R: A Language and Environment for Statistical Computing. 2018. R Foundation for Statistical Computing, Vienna, Austria.

33. Wright RCT, Friman V-P, Smith MCM, Brockhurst MA. Resistance Evolution against Phage Combinations Depends on the Timing and Order of Exposure. mBio 2019; 10 : e01652-19.

34. Mowat E, Paterson S, Fothergill JL, Wright EA, Ledson MJ, Walshaw MJ, et al. Pseudomonas aeruginosa Population Diversity and Turnover in Cystic Fibrosis Chronic Infections. Am J Respir Crit Care Med 2011; 183: 1674-1679.

35. Smith EE, Buckley DG, Wu Z, Saenphimmachak C, Hoffman LR, D'Argenio DA, et al. Genetic adaptation by Pseudomonas aeruginosa to the airways of cystic fibrosis patients. Proc Natl Acad Sci 2006; 103: 8487-8492.

36. Hancock RE, Mutharia LM, Chan L, Darveau RP, Speert DP, Pier GB. Pseudomonas aeruginosa isolates from patients with cystic fibrosis: a class of serum-sensitive, nontypable strains deficient in lipopolysaccharide O side chains. Infect Immun 1983; 42: $170-177$.

37. Huse HK, Kwon T, Zlosnik JEA, Speert DP, Marcotte EM, Whiteley M. Parallel

38. Mahenthiralingam E, Campbell ME, Speert DP. Nonmotility and phagocytic resistance of Pseudomonas aeruginosa isolates from chronically colonized patients with cystic fibrosis. Infect Immun 1994; 62: 596-605.

39. Friman V-P, Ghoul M, Molin S, Johansen HK, Buckling A. Pseudomonas aeruginosa Adaptation to Lungs of Cystic Fibrosis Patients Leads to Lowered Resistance to Phage and Protist Enemies. PLOS ONE 2013; 8: e75380. 
347 41. Chan BK, Sistrom M, Wertz JE, Kortright KE, Narayan D, Turner PE. Phage selection

348 restores antibiotic sensitivity in MDR Pseudomonas aeruginosa. Sci Rep 2016; 6: 26717.

42. Poole K, Zhao Q, Neshat S, Heinrichs DE, Dean CR. The Pseudomonas aeruginosa tonB gene encodes a novel TonB protein. Microbiol Read Engl 1996; 142 ( Pt 6): $1449-1458$.

43. Horvath P, Barrangou R. CRISPR/Cas, the Immune System of Bacteria and Archaea. Science 2010; 327: 167-170.

44. Westra ER, van Houte S, Oyesiku-Blakemore S, Makin B, Broniewski JM, Best A, et al. Parasite Exposure Drives Selective Evolution of Constitutive versus Inducible Defense. Curr Biol 2015; 25: 1043-1049.

45. Doron S, Melamed S, Ofir G, Leavitt A, Lopatina A, Keren M, et al. Systematic discovery of antiphage defense systems in the microbial pangenome. Science 2018; 